\title{
An oncolytic adenovirus expressing interleukin-24 enhances antitumor activities in combination with paclitaxel in breast cancer cells
}

\author{
LIN FANG $^{1 *}$, QIAN CHENG ${ }^{1 *}$, JIN BAI $^{1}$, YING-DONG QI $^{1}$, JUN-JIE LIU $^{2}$, \\ LIAN-TAO LI $^{1}$ and JUN-NIAN ZHENG ${ }^{1,2}$ \\ ${ }^{1}$ Jiangsu Key Laboratory of Biological Cancer Therapy, Xuzhou Medical College; \\ ${ }^{2}$ Laboratory of Urology, Affiliated Hospital of Xuzhou Medical College, Xuzhou, Jiangsu 221002, P.R. China
}

Received April 12, 2013; Accepted August 21, 2013

DOI: $10.3892 / \mathrm{mmr} .2013 .1680$

\begin{abstract}
Oncolytic adenoviruses are a novel class of anticancer treatment, based upon their ability to replicate selectively within malignant cells resulting in cell lysis. The replication-selective adenovirus, ZD55-IL-24, was constructed by harboring an E1B-55 kDa deletion and arming with interleukin-24 (IL-24). The microtubule-stabilizing drug paclitaxel (PTX) exhibits activity in relapsed cancer. In the present study, the synergistic antitumor effects of the combination of PTX and ZD55-IL-24 on breast cancer cells was investigated. The results demonstrated that there were different roles for PTX in the expression of transgenic mRNA and protein. ZD55-IL-24 combined with PTX induced marked growth inhibition of MDA-MB-231 and Bcap-37 cells. PTX increased viral uptake and appeared not to alter the replication of ZD55-IL-24 in breast cancer cells. Annexin V-fluorescein isothiocyanate/propidium iodide staining and the Hoechst 33258 assay indicated that ZD55-IL-24 induced an increase in the number of apoptotic cells when administered in combination with PTX. It was demonstrated that ZD55-IL-24 conjugated with PTX was highly concomitant, and increased proapoptotic proteins levels, activated caspase- $3,-7$ and -9 and downregulated anti-apoptotic proteins. These results suggested that ZD55-IL-24 in combination with PTX exhibited a markedly increased cytotoxic and apoptosis-inducing effect in breast cancer cells. Thus, this chemo-gene-viro therapeutic strategy was demonstrated to be superior to conventional chemotherapy or gene-viro therapy alone.
\end{abstract}

Correspondence to: Dr Jun-Nian Zheng, Jiangsu Key Laboratory of Biological Cancer Therapy, Xuzhou Medical College, 84 Huaihai Road, Xuzhou, Jiangsu 221002, P.R. China

E-mail: fl@xzmc.edu.cn

${ }^{*}$ Contributed equally

Key words: breast cancer, oncolytic adenovirus, interleukin-24, paclitaxel

\section{Introduction}

Breast cancer is one of the most common types of malignant tumors in females and results in high mortality rates (1). Although numerous diagnostic techniques and therapeutic strategies have been identified, metastatic breast cancer remains an incurable disease with little response to current therapeutic approaches (2). Thus, the development of more effective therapeutic strategies is required.

Oncolytic adenoviruses have been proposed as a potential gene therapy vehicle for cancer cells as it enables tumor-selective replication, viral self-spreading and guaranteed efficient expression of transgene in cancer cells without cross-resistance to current treatments $(3,4)$. Recently, numerous modified oncolytic adenoviruses are becoming agents for cancer treatment. This includes tumor-specific promoters which are used to drive the early viral gene, chimeric fibers which are constructed to improve infection efficacy, and viral genes which are deleted to prevent oncogene activation. The human telomerase reverse transcriptase promoter (TERTp) $(5,6)$, hypoxia response elements (HRE)-containing promoter (7) and survivin promoter (8) are present in various cancer cells. Moreover, a human $\alpha$-fetoprotein (AFP) promoter has also been used in AFP-producing cells $(9,10)$ and a cyclooxygenase-2 (Cox 2$)$ promoter $(11,12)$ used in cells overexpressing Cox2. For high infection activity of adenovirus serotype 5 (Ad5), which requires the coxsackie and adenovirus receptor (CAR) localized on the surface of cancer cells, the Ad5/35 chimeric fiber was created $(13,14)$. This chimeric fiber infected cells in a CD46-dependent mechanism. In addition, an RGD-modified fiber has also been used in cancer gene therapy (15).

The initial and most intensively engineered gene deletion, adenoviral ONYX-015 (dl1520), was based on the deletion of E1B-55 kDa, which prevents p53 binding and degradation in normal cells $(16,17)$. An adenovirus deletion mutant $d l 922-947$ (a 24-bp deletion) defective in pRb-binding was engineered in order to improve potency. It has been shown that $d 1922-947$ activity was associated with deregulation of multiple cell cycle checkpoints (18). ZD55 constructed by Professor Liu (Chinese Academy of Sciences, Shanghai, 
China), harbored the E1B-55 kDa deletion and a $B g l \mathrm{II}$ restriction site for carrying the therapeutic gene within the genome. ZD55 has been demonstrated to exhibit efficient activities in tumor therapy (19). One of our previous studies introduced a replication-selective adenovirus with improved potency, ZD55-IL-24. It was constructed by harboring the E1B-55 kDa deletion and arming ZD55 with interleukin-24 (IL-24). IL-24 also termed melanoma differentiation associated gene-7, exhibited multiple antitumor activities, including suppressing growth and inducing the apoptosis of tumor cells without harming normal cells $(20,21)$.

However, efficient treatments usually combine a number of different therapies, including surgical removal, chemotherapy and radiotherapy $(22,23)$. Safety and tumor selectivity of ONYX-015 was demonstrated; however, efficacy was achieved only in combination with chemotherapeutic agents, such as cisplatin or 5-FU in advanced cancer (24). A modest response of $d l 922-947$ was also reported in combination with gemcitabine in pancreatic cancer cells compared with trials using the virus alone $(25,26)$. The microtubule-stabilizing drug paclitaxel (PTX) exhibits activity in relapsed cancer and regimes containing a weekly low-dose PTX are highly effective (27).

The present study aimed to investigate the activities of ZD55-IL-24 in combination with PTX in breast cancer cells. The results suggested that when ZD55-IL-24 was combined with PTX, cancer cell cytotoxicity was markedly increased. PTX increased viral uptake but did not affect the replication of ZD55-IL-24 in breast cancer cells. Notably, it was demonstrated that ZD55-IL-24 conjugated with PTX promoted a markedly stronger cell apoptosis by regulating the intrinsic apoptotic pathway. These results showed that ZD55-IL-24 in combination with PTX exhibited a marked increase in the induced cytotoxic and apoptotic effect in breast cancer cells. This chemo-gene-viro therapeutic strategy is superior to conventional chemotherapy or gene-viro therapy alone.

\section{Materials and methods}

Cell culture and adenoviral construction. MDA-MB-231 and Bcap-37 human breast cancer cell lines were obtained from the Institute of Biochemistry and Cell Biology, Shanghai Institute for Biological Sciences, Chinese Academy of Sciences (Shanghai, China). The (HEK)-293 human embryonic kidney cell lines were obtained from Microbix Biosystems Inc. (Toronto, ON, Canada). MDA-MB-231 was cultured in Leibovitz's L-15 medium (Life Technologies, Carlsbad, CA, USA) with fetal bovine serum (FBS) to a final concentration of 10\%. Bcap-37 was cultured in RPMI-1640 medium (Life Technologies) with FBS to a final concentration of $10 \%$. HEK-293 was cultured in Dulbecco's minimal essential medium (Life Technologies) with FBS to a final concentration of $10 \%$. All media were supplemented with $4 \mathrm{mM}$ glutamine, $100 \mathrm{U} / \mathrm{ml}$ penicillin, and $100 \mathrm{mg} / \mathrm{ml}$ streptomycin. All cells in the experiment were cultured under a $5 \% \mathrm{CO}_{2}$ humidified atmosphere at $37^{\circ} \mathrm{C}$.

The replication-selective adenovirus, ZD55-IL-24, had been described previously and was constructed by harboring the E1B-55 kDa deletion and arming with IL-24 (21). ZD55-EGFP was also constructed as a control virus, which carried enhanced green fluorescent protein (EGFP) as a reporter gene. Large-scale preparations of these adenoviruses were produced by ultracentrifugation (Optima L-100XP, Beckman,USA) on cesium chloride. Paclitaxel (PTX) was purchased from Nanjing Pharmaceutical Factory Co., Ltd. (Nanjing, Jiangsu, China). A final concentration of $0.25 \mu \mathrm{g} / \mathrm{ml}$ was used as the treatment dose based on preliminary experiments.

Reverse transcription-PCR. Bcap-37 cells were treated with $0.25 \mu \mathrm{g} / \mathrm{ml}$ PTX, 10 multiplicity of infection (MOI) ZD55-EGFP; 10 MOI ZD55-IL-24; 10 MOI ZD55-EGFP and $0.25 \mu \mathrm{g} / \mathrm{ml}$ PTX; or 10 MOI ZD55-IL-24 and $0.25 \mu \mathrm{g} / \mathrm{ml}$ PTX. After $48 \mathrm{~h}$, total RNA was extracted using TRIzol reagent (Invitrogen Life Technologies, Carlsbad, CA, USA). cDNA was obtained from the total RNA using the One Step RT-PCR kit (Promega, Madison, WI, USA) according to the manufacturer's instructions. Primers used for the quantification of E1A, IL-24 and glyceraldehyde 3-phosphate dehydrogenase (GAPDH) mRNA were as follows: Sense: 5'-ATTATCTGCCACGGAGGTGTT-3' and antisense: 5'-CAAAGGTTGCCCAGACTC-3' for E1A; sense: 5'-CCCGGTCGACATGAATTTTCAACAGAGG-3' and antisense: 5'-GGGTGGATCCTCAGAGCTTGTAGAATT TCTGC-3'; for IL-24; and sense: 5'-TCCATGA CAACTTTGGTATC-3' and antisense: 5'-TTCAGCTCAGG GATGACCTT-3' for GAPDH.

Western blot analysis. Following treatment, Bcap-37 cells were scraped into $200 \mu \mathrm{l}$ lysis buffer [containing $150 \mathrm{mmol} / \mathrm{l}$ $\mathrm{NaCl}, 50 \mathrm{mmol} / \mathrm{l}$ Tris ( $\mathrm{pH} 7.5$ ), $0.05 \%$ sodium dodecyl sulphate (SDS) and 1\% Triton X-100] and sonicated on ice. Protein $(50 \mu \mathrm{g})$ was electrophoresed on SDS-polyacrylamide gels and transferred onto a nitrocellulose filter by semi-dry blotting. The following antibodies were used: Mouse anti-E1A (Santa Cruz Biotechnology, Inc., Santa Cruz, CA, USA), rabbit anti-IL-24, rabbit anti-(cleaved) caspase-3, -7 and -9 , rabbit anti-(cleaved) poly ADP ribose polymerase (PARP), rabbit anti-B-cell lymphoma-extra large (BCL-XL), rabbit anti-myeloid leukemia cell differentiation protein (MCL-1) rabbit anti-Bcl-2-associated X protein (BAX; Cell Signaling Technology, Inc., Danvers, MA, USA), and mouse anti- $\beta$-actin (Santa Cruz Biotechnology, Inc.). Detection was performed by AP-conjugated antibodies and NBT/BCIP substrate (Promega, Madison, WI, USA).

Cell viability assay. For the cell viability assay, MDA-MB-231 and Bcap-37 breast cancer cells were plated on 96-well plates at a density of $1 \times 10^{4}$ cells/well 1 day prior to virus infection. Cells were then infected with phosphate-buffered saline; $0.25 \mu \mathrm{g} / \mathrm{ml}$ PTX; ZD55-EGFP; ZD55-IL-24; ZD55-EGFP and $0.25 \mu \mathrm{g} / \mathrm{ml}$ PTX; or ZD55-IL-24 and $0.25 \mu \mathrm{g} / \mathrm{ml}$ PTX, different adenoviruses were used at an MOI of 10. On days 1, 2, 3 and 4 following treatment, cell viability was determined by an MTT assay according to the manufacturer's instructions at the indicated time. Cells were also infected with ZD55-EGFP or ZD55-IL-24 at doses of different MOIs with different concentrations of PTX $(0.1 \mathrm{MOI}+0.01 \mu \mathrm{g} / \mathrm{ml}, 1 \mathrm{MOI}+0.05 \mu \mathrm{g} / \mathrm{ml}$, $10 \mathrm{MOI}+0.25 \mu \mathrm{g} / \mathrm{ml}$ or $50 \mathrm{MOI}+0.5 \mu \mathrm{g} / \mathrm{ml})$. Cell viability was detected 4 days following infection. In each treatment group, eight wells were measured for cell viability. All cell 
viability assays were conducted in triplicate. Representative results are shown unless otherwise stated.

Flow cytometric analysis. MDA-MB-231 and Bcap-37 cells were incubated with $0.25 \mu \mathrm{g} / \mathrm{ml}$ PTX for $24 \mathrm{~h}$, followed by infection with 10 MOI ZD55-EGFP for $2 \mathrm{~h}$. On day 0,1 or 2 following infection, EGFP expression was observed under a fluorescence microscope. The cells were then harvested $48 \mathrm{~h}$ after infection and the proportion of EGFP-positive cells was determined by flow cytometry (FACS-Canto II instrument, BD, UK), acquiring 20,000 events per sample from duplicate wells using propidium iodide (PI) to exclude dead cells. The analysis was conducted using Cell Quest software (BD Biosciences, Franklin Lakes, NJ, USA).

Viral replication assay. For viral replication assays, cells were placed in 6-well plates at a density of $5 \times 10^{5}$ cells/well. The cells were treated with $0.25 \mu \mathrm{g} / \mathrm{ml}$ PTX and $24 \mathrm{~h}$ subsequent to this, were infected with ZD55-IL-24 or ZD55-EGFP at an MOI of $10 \mathrm{pfu} / \mathrm{cell}$. Cells infected with ZD55-IL-24 or ZD55-EGFP but not added to PTX served as controls. At $0 \mathrm{~h}$ (the beginning of infection) and $48 \mathrm{~h}$ following infection, the cells were harvested, lysed by three cycles of freeze/thaw at $-80^{\circ} \mathrm{C}$ and $37^{\circ} \mathrm{C}$ and serial dilutions of the lysates were subsequently tittered on HEK293 cells with tissue culture infectious dose $50\left(\right.$ TCID $\left._{50}\right)$ methods, as described previously (28).

Cell apoptosis analysis. MDA-MB-231 and Bcap-37 cells were infected with $0.25 \mu \mathrm{g} / \mathrm{ml}$ PTX; 10 MOI ZD55-EGFP; $10 \mathrm{MOI}$ ZD55-IL-24; 10 MOI ZD55-IL-24 and $0.25 \mu \mathrm{g} / \mathrm{ml} \mathrm{PTX;} \mathrm{or}$ ZD55-IL-24 and $0.25 \mu \mathrm{g} / \mathrm{ml}$ PTX. The apoptotic cells were assayed with Annexin V-fluorescein isothiocyanate (FITC) and propidium iodide (Annexin V-FITC Apoptosis Detection Kit, KeyGen Biotech, Nanjing, China) under a fluorescence microscope after $48 \mathrm{~h}$. The fluorescence signal in cells probed by Annexin V-FITC was green and that probed by PI was red. The number of apoptotic cells was counted in five fields of vision chosen at random. The average value was calculated as the rational data.

Hoechst 33258 staining. Cells were treated with drugs or adenovirus as described above for the fluorescence microscopy analysis. Following 2 days, cells were fixed with $4 \%$ paraformaldehyde and stained with Hoechst 33258 (Apoptotic Cell Hoechst 33258 Detection kit, KeyGen Biotech) for $10 \mathrm{~min}$ at room temperature. Fluorescence was visualized with a Nikon standard fluorescence microscope (Ti-U, Nikon, Japan). The number of cells with nuclei condensation and fragmentation were counted.

Statistical analysis. Data are expressed as the mean \pm SD unless otherwise stated. Results were compared for statistical significance by a t-test. $\mathrm{P}<0.05$ and $\mathrm{P}<0.01$ were considered to indicate a statistically significant difference.

\section{Results}

PTX results in an increase in E1A and IL-24 mRNA but not protein expression levels in breast cancer cells. To investigate the effect of PTX on adenovirus gene expression, the Bcap-37 human breast cancer cell line was treated with $0.25 \mu \mathrm{g} / \mathrm{ml}$ PTX, infected with 10 MOI ZD55-EGFP or ZD55-IL-24, or ZD55-EGFP or ZD55-IL-24 in combination with PTX. RT-PCR analysis showed an increase in E1A mRNA level $48 \mathrm{~h}$ following infection with ZD55-EGFP or ZD55-IL-24 in combination with PTX; and an IL-24 mRNA level in ZD55-IL-24 plus PTX group (Fig. 1A and B). The quantity of E1A mRNA in the ZD55-EGFP + PTX group was 1.28-fold greater than that in the ZD55-EGFP group, 2.23-fold greater than that in the ZD55-IL-24 + PTX group than that for the ZD55-IL-24 group in the Bcap-37 cells. The quantity of IL-24 mRNA for the ZD55-IL-24 + PTX group was 1.49-fold greater than that in the ZD55-IL-24 group in Bcap-37 cancer cells. There was no E1A and IL-24 mRNA expression in the PTX group. These results indicated that PTX enhanced adenoviral gene mRNA expression.

The expression levels of associated proteins were also investigated. Western blot analysis was performed when Bcap-37 cells had been treated as previously described. Cell lysates were harvested after $48 \mathrm{~h}$. As shown in Fig. 1C, no increase in protein levels of E1A or IL-24 were observed in any oncolytic adenovirus plus PTX treatment group. The control PBS and PTX groups exhibited no E1A and IL-24 expression as expected. These results indicated that PTX increased the transgenic mRNA but not protein expression mediated by oncolytic adenoviruses defective in p53-binding (E1B-55 kDa deletion).

Administration of oncolytic adenovirus in combination with PTX induces increased cytotoxicity in breast cancer cells compared with that in the groups with virus alone. To investigate whether breast cancer cells were able to be sensitized to PTX by oncolytic adenoviruses, $0.25 \mu \mathrm{g} / \mathrm{ml}$ PTX was added with 10 MOI ZD55-EGFP or ZD55-IL-24 in MDA-MB-231 and Bcap-37 cells. Cell cytotoxicity was markedly increased in all tested cell lines treated with ZD55-EGFP or ZD55-IL-24 in combination with PTX compared with that of the control groups (Fig. 2A and B). In addition, we also wanted to confirm whether the strong synergistic cell cytotoxic effect was viral dose-dependent. MDA-MB-231 and Bcap-37 cells were infected with ZD55-EGFP or ZD55-IL-24 at MOIs of 0.1, 1, 10, 50 combined with or without varying concentrations of PTX. Cell viability was also detected following 4 days of infection. The most potent synergistic responses were observed in cells with $0.25 \mu \mathrm{g} / \mathrm{ml}$ PTX with $10 \mathrm{MOI}$ adenovirus ZD55-IL-24 infection (Fig. 2C and D). Moreover, ZD55-IL-24 plus PTX exhibited higher cytotoxicity than ZD55-EGFP plus PTX in these cells. In Bcap-37 cells, the difference between 10 MOI and 50 MOI ZD55-IL-24 combined with PTX and the PTX control group was significant $\left({ }^{*} \mathrm{P}<0.05\right)$. In MDA-MB-231 cells, the difference between the 10 MOI ZD55-IL-24 combined with PTX and the PTX group was significant $\left({ }^{* *} \mathrm{P}<0.01\right)$. In conclusion, oncolytic adenoviruses and PTX increased cell cytotoxicity and to a certain extent, oncolytic adenoviruses enhanced the sensitivity of breast cancer cells to PTX.

PTX increases viral uptake in breast cancer cells and viral replication is not altered in response to PTX. To deter- 
A

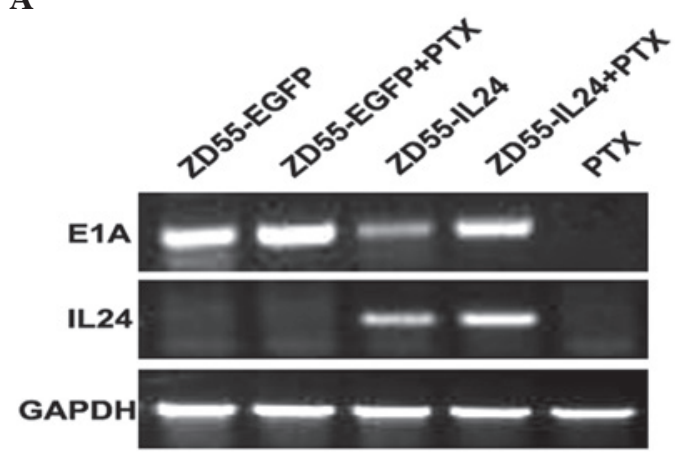

B

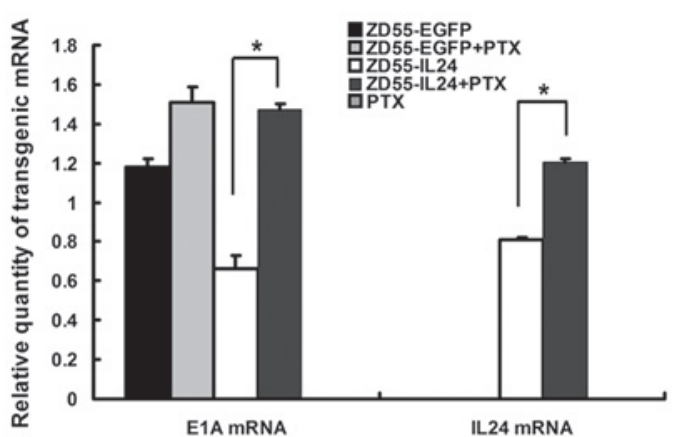

C

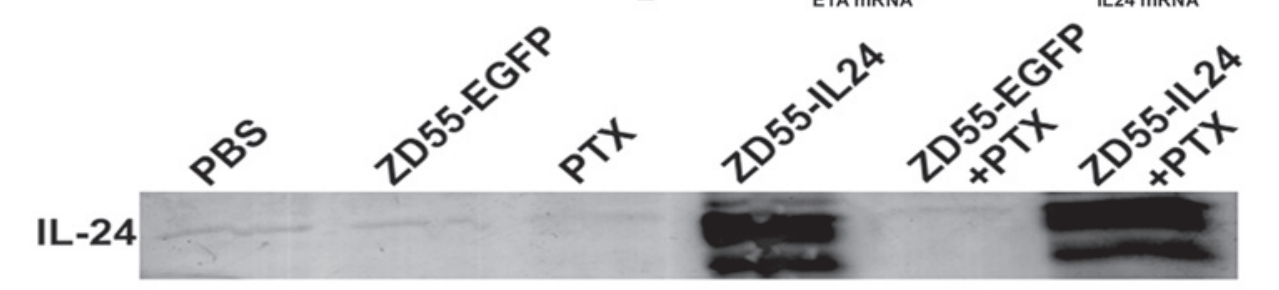

E1A

$\beta$-actin

Figure 1. Transgenic mRNA and protein expression levels in Bcap-37 cells following administration with oncolytic adenovirus in combination with paclitaxel (PTX). (A) RT-PCR analysis of E1A and interleukin-24 (IL-24) transcripts were performed at $48 \mathrm{~h}$ following infection. Glyceraldehyde 3-phosphate dehydrogenase was used as the inner control. (B) Quantification of mRNA expression shown in (A). ${ }^{*}<<0.05$. (C) Cell lysates were harvested at $48 \mathrm{~h}$ following infection as indicated and detected by anti-E1A and anti-IL24 antibodies. $\beta$-actin was used as a control to show input.
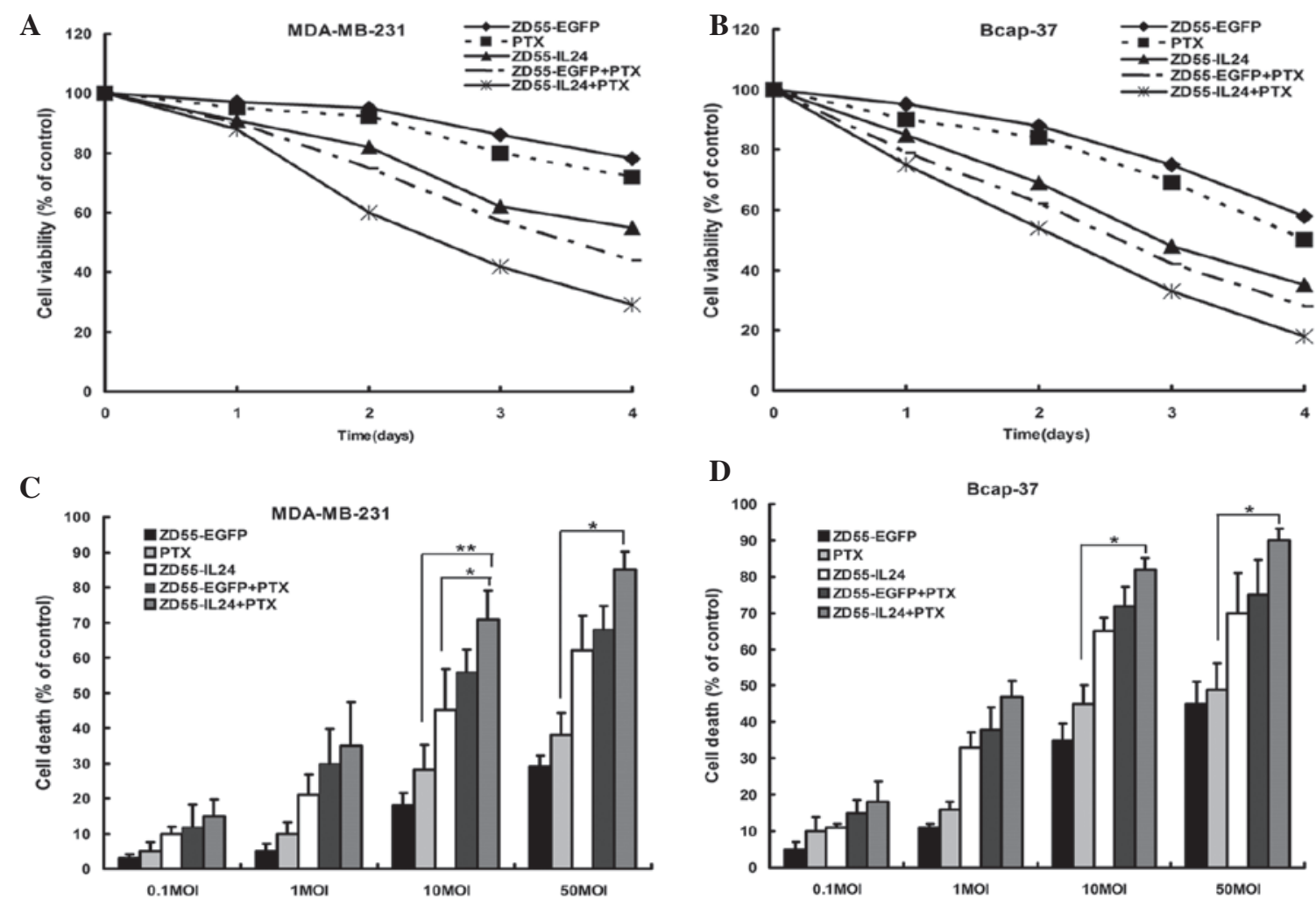

Figure 2. The effects of adenoviruses combined with paclitaxel (PTX) on growth of breast cancer cells. (A) MDA-MB-231 and (B) Bcap-37 cells were infected with phosphate-buffered saline (PBS), $0.25 \mu \mathrm{g} / \mathrm{ml}$ PTX, ZD55-enhanced green fluorescent protein (EGFP), ZD55-interleukin-24 (IL-24), ZD55-EGFP $+0.25 \mu \mathrm{g} / \mathrm{ml}$ PTX or ZD55-IL-24 $+0.25 \mu \mathrm{g} / \mathrm{ml}$ PTX. Cell viability of these cells was measured by MTT assay. The data were expressed as a percentage of the PBS control group. (C) MDA-MB-231 and (D) Bcap-37 cells were infected with ZD55-EGFP or ZD55-IL-24 at different MOIs of 0.1, 1, 10 and $50 \mathrm{pfu} / \mathrm{cell}$ combined with different concentrations or no PTX. Cell viability was analyzed 4 days following treatment. ${ }^{*} \mathrm{P}<0.05$ and ${ }^{* * *} \mathrm{P}<0.01$, compared with the drug or viral group alone. 


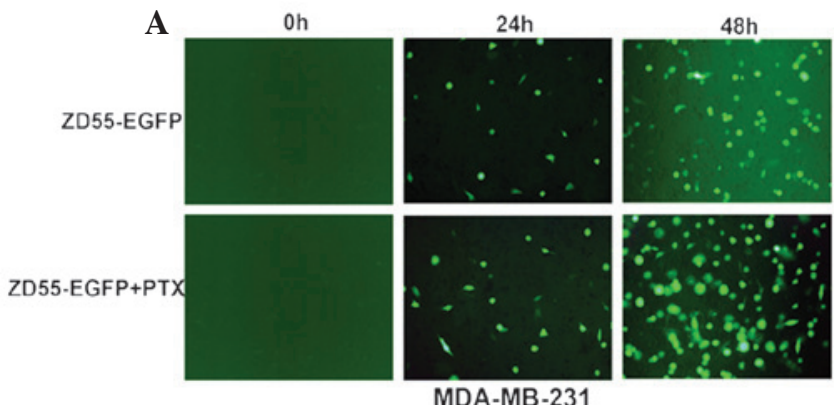

MDA-MB-231
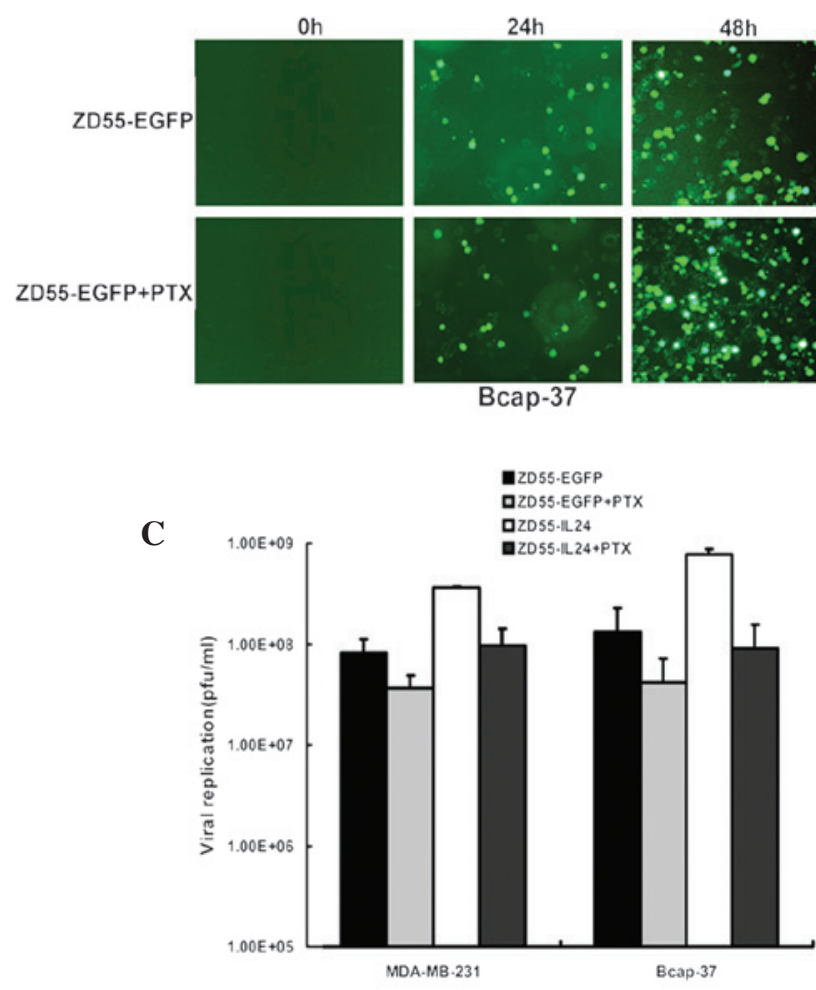

Figure 3. Virus uptake and replication when combined with paclitaxel (PTX) in MDA-MB-231 and Bcap-37 cells. (A) Fluorescent microscopic observation of tumor cells infected with ZD55-enhanced green fluorescent protein (EGFP) virus. Cells were infected at a multiplicity of infection (MOI) of $10 \mathrm{pfu} / \mathrm{cell}$ and then detected on days 0,1 and 2 following infection. On day 2, a cytopathic effect was observed in the tumor cells. Moreover, on day 2, the majority of the tumor cells became round, scraped from the dish or dead. (B) Viral uptake is increased by PTX in breast cancer cell lines. Cells were treated with $0.25 \mu \mathrm{g} / \mathrm{ml}$ PTX for $24 \mathrm{~h}$ prior to infection with the replicating EGFP-expressing ZD55-EGFP virus at an MOI of 10 in MDA-MB-231 and Bcap-37 cells. The percentages of EFGP-positive cells were determined by flow cytometry $48 \mathrm{~h}$ following infection. Mock-treated cells were infected with ZD55-EGFP without the addition of PTX. Data are presented as the mean $\pm \mathrm{SD}$. ${ }^{*} \mathrm{P}<0.05$ and ${ }^{* *} \mathrm{P}<0.01$, compared with mock. Original magnification, $\mathrm{x} 200$. (C) Viral replication is attenuated by PTX, but not early E1A gene expression. MDA-MB-231 and Bcap-37 cells were incubated with $0.25 \mu \mathrm{g} / \mathrm{ml} 24$ h prior to infection with adenoviruses ZD55-EGFP or ZD55-interleukin-24 (IL-24) at 10 MOI. Cells infected with ZD55-IL-24 or ZD55-EGFP only served as mocks. At the indicated time, cells were lysed and viral titre was quantified by the tissue culture infectious dose 50 (TCID50) assay. Viral production was expressed as pfu/ml.

mine whether the observed synergy was due to increased permissiveness to viral infection in the presence of PTX, MDA-MB-231 and Bcap-37 cells were infected with an EGFP-expressing virus (ZD55-EGFP) to monitor viral uptake. On day 0,1 or 2 following infection, EGFP expression was observed by fluorescence microscopy (Fig. 3A). The EGFP expression level was low $24 \mathrm{~h}$ following infection, but was significantly increased after $48 \mathrm{~h}$. Moreover, EGFP expression in these cells was increased in ZD55-EGFP combined with PTX compared with ZD55-EGFP alone. Furthermore, the expression of EGFP tagged virus in these cells was determined by flow cytometry analysis. The cells were harvested $48 \mathrm{~h}$ following infection and the proportion of EGFP-positive cells was determined by acquiring 20,000 events per sample from duplicate wells using PI to exclude dead cells. As shown in Fig. 3B, viral uptake increased with the administration of PTX in the two breast cancer cell lines. The difference was significant between combinations of virus and PTX and mock (virus without PTX). The number of EGFP-positive cells in ZD55-EGFP combined with PTX was 5-fold that in ZD55-EGFP infected MDA-MB-231 cells, and 3-fold that in Bcap-37 cells.

The study also aimed to detect the potential drug-induced effects of a combination treatment on factors of the viral life cycle, such as replication. As shown in Fig. 3C, viral replica- tion was high after $48 \mathrm{~h}$ at a MOI of 10 for ZD55-EGFP and ZD55-IL-24 infected MDA-MB-231 and Bcap-37 cells. When cells were pretreated with PTX at $0.25 \mu \mathrm{g} / \mathrm{ml}$, viral replication was marginally attenuated in the two cell lines. The expression of E1A was also detected in the treatment group and there was no significant change in the presence of PTX (data not shown). The replication of the virus was closely associated with the expression of E1A. The results also demonstrated that the involvement of E1A in replication and was consistent with the fact that PTX did not increase protein expression mediated by oncolytic adenovirus defective in p53-binding (E1B-55 kDa deletion) (Fig. 1C).

Oncolytic adenovirus in combination with PTX induced increased apoptosis in breast cancer cells. Apoptosis in MDA-MB-231 and Bcap-37 cells were infected with ZD55-EGFP or ZD55-IL-24, PTX, or a combination of both. For the early apoptosis assay, cells were incubated with Annexin V-FITC following treatment for $48 \mathrm{~h}$. As shown in Fig. 4A, there was a marked cytopathic effect and cells became rounded. The number of green fluorescent cells was greatest in the ZD55-IL-24+PTX group compared with the other groups. As ZD55-EGFP itself expressed green fluorescence and due to the difficulty in distinguishing EGFP with FITC, apoptosis in the ZD55-EGFP or ZD55-EGFP+PTX groups 
A

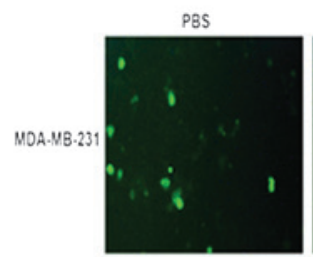

PBS

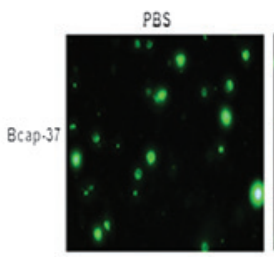

B

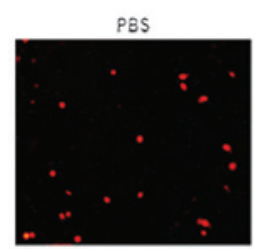

2055.1624

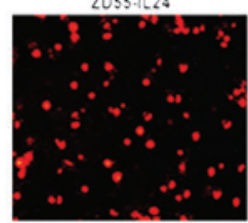

PTX

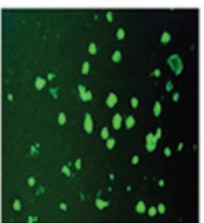

PTX

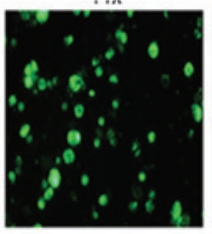

2055. 11.24

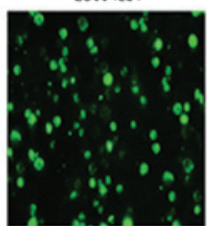

$2055 \cdot 1.24$

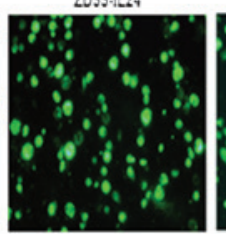

2055:.L24.PTX

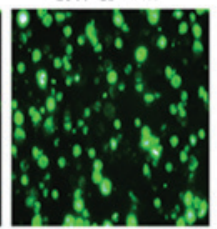

$2055 \cdot 1.24+P T X$

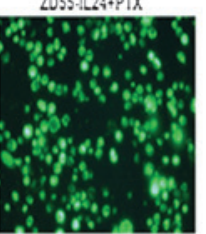

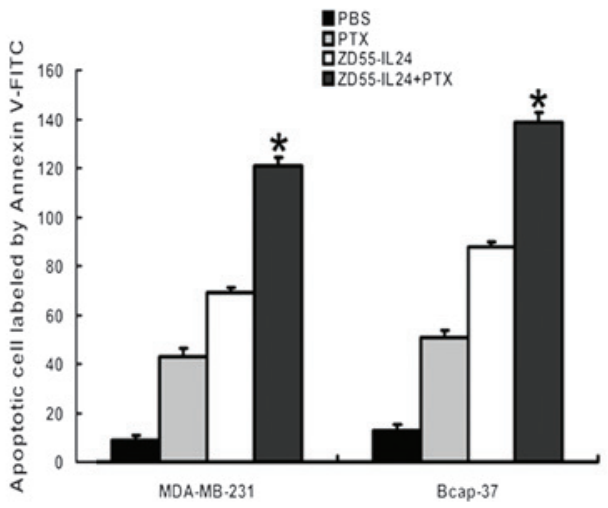

PBS

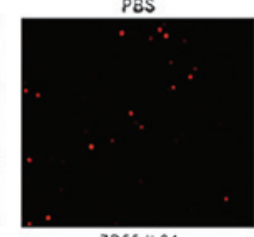

2055.1L24

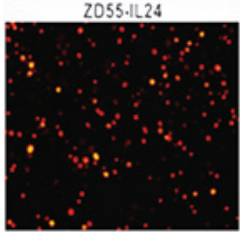

aPB

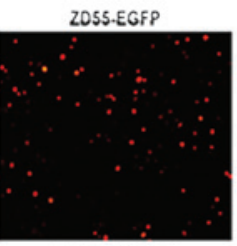

ZOS5.EGFP+PTX

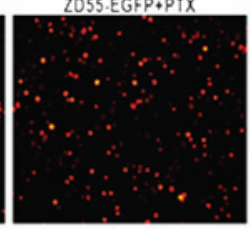

8cap. -37
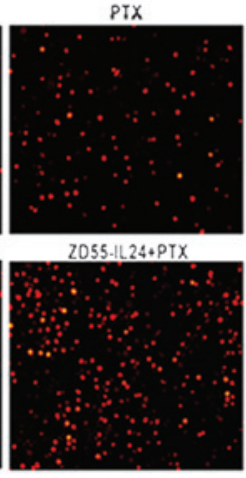

MOA.MB.231

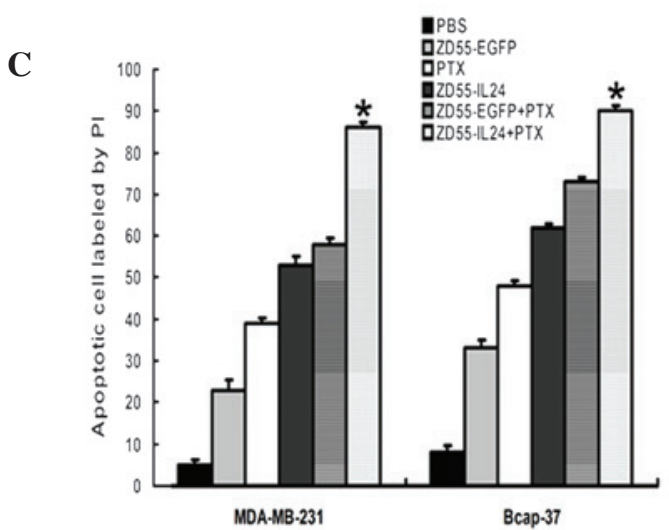

Figure 4. The apoptotic cells were induced by virus or paclitaxel (PTX) treatment. MDA-MB-231 and Bcap-37 cells placed into 6-well plates were treated with virus, drug or combination. (A) The cells were stained with Annexin V-fluorescein isothiocyanate (FITC), the graph represents the statistical analysis of the FITC-positive cells. (B and C) After 2 days, the cells were stained with propidium iodide. * $\mathrm{P}<0.05$, compared with the virus or PTX group alone. The fluorescence signal was obtained under a fluorescence microscope. Original magnification, x200.

was not analyzed in this assay. The results from the analysis of apoptosis using PI were similar to that when analyzed with FITC (Fig. 4B and C). A combination of ZD55-IL-24 and PTX resulted in large scale cell apoptosis. The quantization of positive cells was analyzed by Image-J software. The fluorescence signal in cells probed by Annexin V-FITC (green) and PI (red) was obtained by fluorescence microscopy. The number of apoptotic cells was counted in five fields of vision chosen at random. The average value was calculated as the rational data.

Oncolytic adenovirus in combination with PTX induces apoptosis in breast cancer cells via a mitochondria-mediated pathway. The apoptosis of cells was also analyzed by
Hoechst 33258 staining following treatment (Fig. 5A). Nuclear fragmentation and chromatin clumping were observed in cells in the ZD55-IL-24 plus PTX group. The number of cells with nuclear fragmentation was greatest in the ZD55-IL-24 plus PTX group (Fig. 5B). The components of the apoptosis signaling cascade were investigated by immunoblotting in Bcap-37 cells. As shown in Fig. 5C, BAX, cleaved caspase-3, -7 and -9 , and PARP were upregulated and the anti-apoptotic proteins BCL-XL and MCL-1 were decreased following treatment with oncolytic adenovirus, PTX or a combination of the two. It was observed that the ZD55-IL-24 plus PTX group exhibited a higher expression level of pro-apoptotic protein (BAX), lower anti-apoptotic protein (BCL-XL and MCL-1) and higher downstream active caspase family members. These 
A
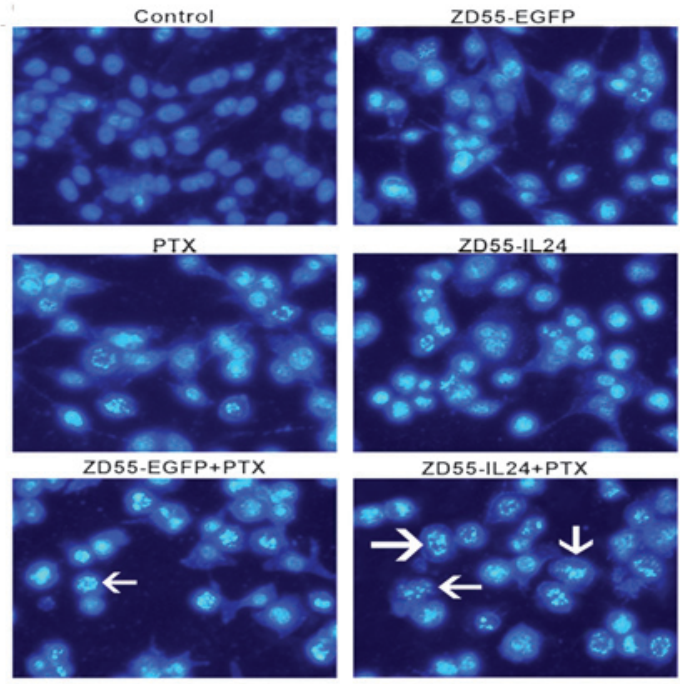

MDA-MB-231

$\mathbf{B}$
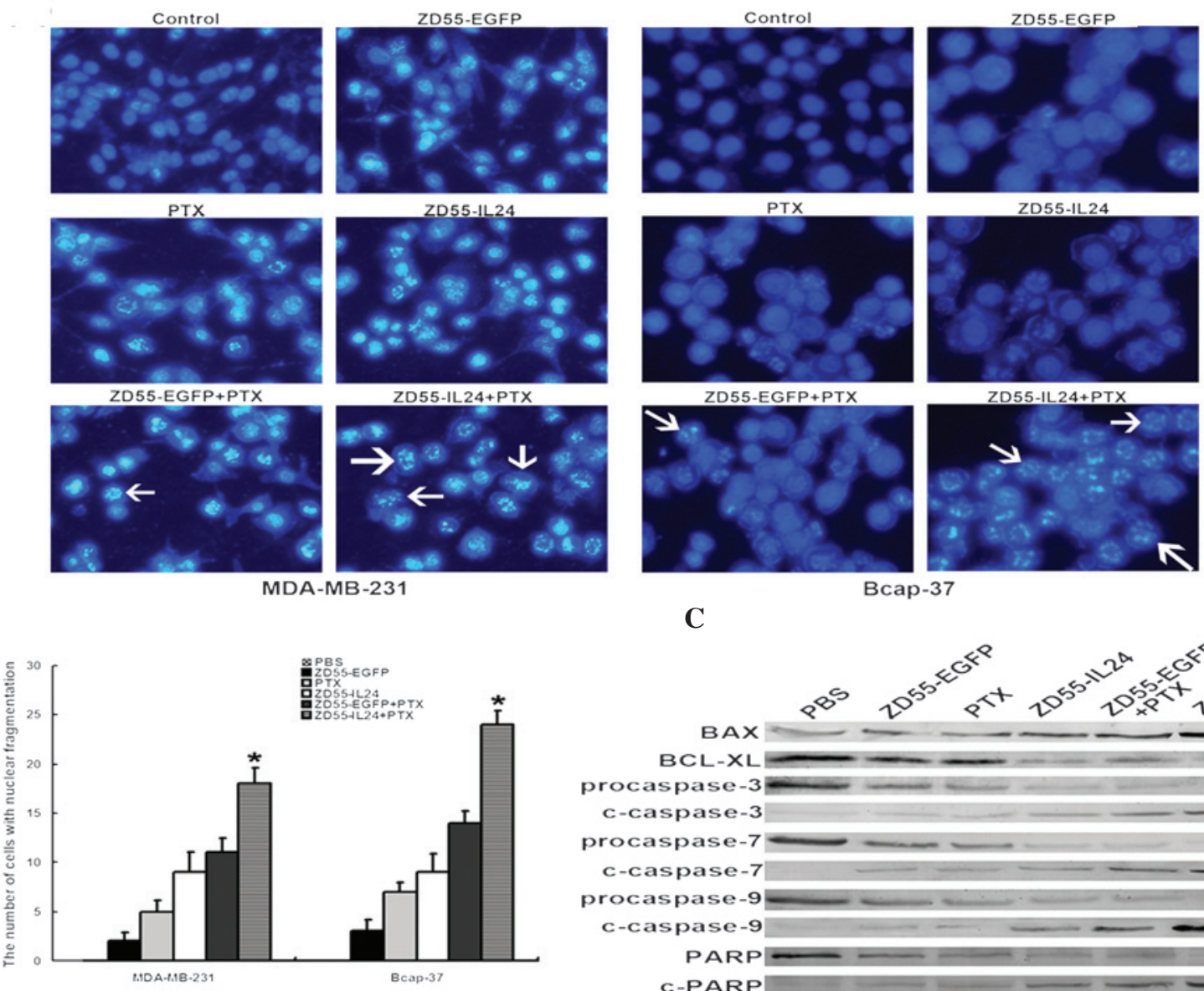

C

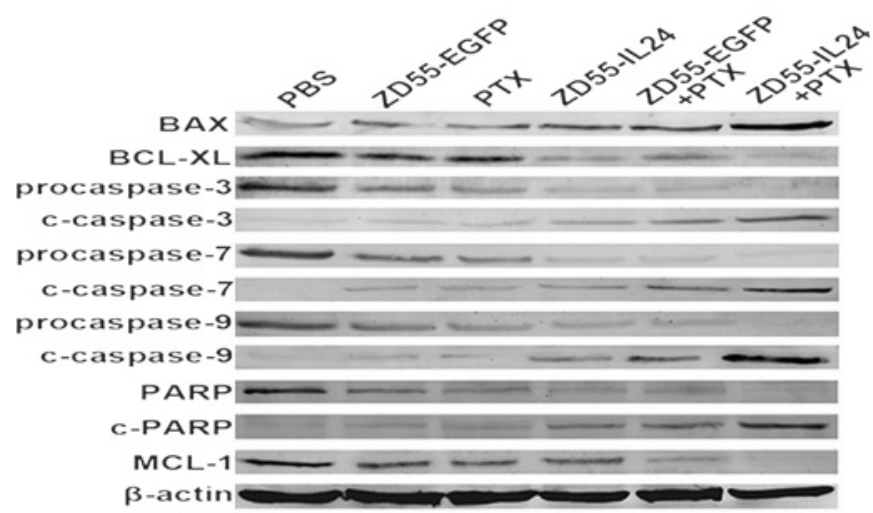

Figure 5. (A) Apoptosis of cells confirmed by Hoechst 33258 staining following treatment. Nuclear fragmentation and chromatin clumping were shown in cells infected with ZD55-IL-24 followed by paclitaxel (PTX). (B) Number of cells with nuclear fragmentation was the greatest in the ZD55-IL-24 plus PTX group than that in other groups. (C) Bcl-2-associated X protein (BAX), cleaved caspase-3, -7 and -9 and poly ADP-ribose polymerase (PARP) were upregulated and the anti-apoptotic proteins B-cell lymphoma-extra large (BCL-XL) and myeloid leukemia cell differentiation protein (MCL-1) were decreased following treatment with oncolytic adenovirus, PTX or a combination of the two.

results showed that ZD55-IL-24 in combination with PTX induced cell apoptosis efficiently and exhibited enhanced anti-tumor activities in breast cancer cells.

\section{Discussion}

The incidence of breast carcinoma has increased annually and the prognosis for this cancer is remains poor regardless of the improved treatment options during the past decades (29). Traditional treatment, such as surgery, chemotherapy or radiotherapy are not breakthrough therapies and are associated with significant side effects, thus the development of novel potent curative treatments is required. Oncolytic adenoviruses provide numerous advantages in cancer therapy (30) due to selective and efficient replication in tumor cells, which reduced the therapeutic dose. The viruses replicate within and lyse tumor cells, which leads to cell death. Novel progeny viruses released from host cells infect neighboring cells continually and ultimately eliminate tumor cells. The combination of viral and gene therapy developed into the novel strategy of gene-viro therapy, in which therapeutic genes amplified abundantly with adenovirus replication and functioned as antitumor agents $(31,32)$. Recently, it has been demonstrated that the combination of replication-selective oncolytic adenoviruses with chemotherapeutic agents is a promising cancer therapy (33).

PTX was approved as a novel drug for the treatment of ovarian cancer by the FDA in 1992 (34). Numerous studies have confirmed that a variety of proteins are related to paclitaxel-induced apoptosis such as Bcl-2 protein family of Raf-1, caspase-3, PARP, P34/cdc2, p53, and p27 (35-38). Like most chemotherapeutic drugs, paclitaxel exhibits side effects, and the treatment efficacy is attenuated when the dosage is reduced. Therefore, utilization of lower doses of PTX combined with other treatments may achieve similar or improved therapeutic effects without increasing the side effects.

The results of the present study suggested that oncolytic IL-24 armed adenovirus with the E1B-55 kDa deletion (ZD55-IL-24) exhibited significant activity when combined with low doses $(0.25 \mu \mathrm{g} / \mathrm{ml})$ of the microtubule-stabilizing drug, PTX, through the mitochondria-mediated pathway in breast cancer cells. The mRNA and protein expression were investigated when cells had been infected with ZD55-EGFP or ZD55-IL-24 in combination with PTX. The mRNA level 
of E1A and transgenes (EGFP and IL-24) in oncolytic adenoviruses increased; however, the protein expression did not increase accordingly (Fig. 1C). These results demonstrated the different roles of PTX in the expression of oncolytic adenovirus transgenic mRNA and protein. A possible mechanism explaining this result is that PTX is a microtubule-stabilizing drug and adenoviruses require microtubules for nuclear transport. Further studies are required to determine the contribution of PTX in adenovirus mediated cancer therapy.

It was also demonstrated that the cell cytotoxic efficacy of ZD55-IL-24 combined with PTX was greater than that with virus or drug treatment alone. The synergistic cell cytotoxic effect of the virus plus PTX groups was virus dose-dependent. ZD55-IL-24 and PTX may have a synergistic effect depending on different mechanisms. ZD55-IL-24 promoted the sensitivity of cells to cytotoxic drugs. The results also demonstrated that although PTX increased viral uptake, viral replication was not affected in response to PTX in breast cancer cells. As adenoviral infection and replication were different biological behaviors, cell surface receptors (e.g. CAR) were required for infection and key viral genes (e.g. E1A, the first viral gene expressed) were important for viral replication. Annexin V-FITC/PI staining and the Hoechst 33258 assay indicated that ZD55-IL-24 induced greater cell apoptosis when combined with PTX. Furthermore, the possible mechanism was investigated. ZD55-IL-24 expressed IL-24 with high efficiency, which was concomitant with increased levels of pro-apoptotic proteins, activated caspase-3, -7 and -9 and downregulated anti-apoptotic proteins. These results showed that ZD55-IL-24 conjugated with PTX exhibited a markedly increased cytotoxic and apoptotic effect in breast cancer cells.

In conclusion, replication-selective oncolytic adenoviruses carrying the IL-24 gene, ZD55-IL-24, significantly enhanced the effects of PTX on the suppression of MDA-MB-231 and Bcap-37 cell proliferation and induced efficient cell apoptosis. PTX did not affect the protein expression of the therapeutic genes and the replication of ZD55-IL-24 in breast cancer cells. Thus, this study suggested that gene-viro therapy combined with chemotherapy represent a potent approach for breast cancer therapy.

\section{Acknowledgements}

This study was supported by grants from the National Natural Science Foundation of China (grant nos. 81101702, 81071854 and 30972976); the Science and Technology Department of Jiangsu province (grant nos. BK2011207, BK2009091 and BK2009089); the College Science and Technology Foundation of Jiangsu province (grant nos. 12KJA320001 and 11KJA320002); the Science Foundation of Xuzhou Medical College (grant no. 2010KJZ04); and the Science and Technology Department of Xuzhou (no. XF11C061).

\section{References}

1. Krychman ML and Katz A: Breast cancer and sexuality: multi-modal treatment options. J Sex Med 9: 5-13, 2012.

2. Haas JS, Liang SY, Hassett MJ, Shiboski S, Elkin EB and Phillips KA: Gene expression profile testing for breast cancer and the use of chemotherapy, serious adverse effects, and costs of care. Breast Cancer Res Treat 130: 619-626, 2011.
3. Choi JW, Lee JS, Kim SW and Yun CO: Evolution of oncolytic adenovirus for cancer treatment. Adv Drug Deliv Rev 64: 720-729, 2012

4. Huang JH, Zhang SN, Choi KJ, et al: Therapeutic and tumor-specific immunity induced by combination of dendritic cells and oncolytic adenovirus expressing IL-12 and 4-1BBL. Mol Ther 18: 264-274, 2010.

5. Hashimoto Y, Tazawa H, Teraishi F, et al: The hTERT promoter enhances the antitumor activity of an oncolytic adenovirus under a hypoxic microenvironment. PLoS One 7: e39292, 2012.

6. Fang L, Pu YY, Hu XC, et al: Antiangiogenesis gene armed tumor-targeting adenovirus yields multiple antitumor activities in human HCC xenografts in nude mice. Hepatol Res 40: 216-228, 2010.

7. Cho WK, Seong YR, Lee YH, et al: Oncolytic effects of adenovirus mutant capable of replicating in hypoxic and normoxic regions of solid tumor. Mol Ther 10: 938-949, 2004.

8. Ulasov IV, Zhu ZB, Tyler MA, et al: Survivin-driven and fiber-modified oncolytic adenovirus exhibits potent antitumor activity in established intracranial glioma. Hum Gene Ther 18: 589-602, 2007.

9. Kim KI, Park JH, Lee YJ, et al: In vivo bioluminescent imaging of $\alpha$-fetoprotein producing hepatocellular carcinoma in diethylnitrosamine-treated mouse using recombinant adenoviral vector. J Gene Med 14: 513-520, 2012.

10. Kwon OJ, Kim PH, Huyn S, Wu L, Kim M and Yun CO: A hypoxia- and $\alpha$-fetoprotein-dependent oncolytic adenovirus exhibits specific killing of hepatocellular carcinomas. Clin Cancer Res 16: 6071-6082, 2010.

11. Nakagawa T, Tanaka H, Shirakawa T, et al: Cyclooxygenase 2 promoter-based replication-selective adenoviral vector for hypopharyngeal cancer. Arch Otolaryngol Head Neck Surg 135: 282-286, 2009.

12. Bauerschmitz GJ, Guse K, Kanerva A, et al: Triple-targeted oncolytic adenoviruses featuring the cox 2 promoter, E1A transcomplementation, and serotype chimerism for enhanced selectivity for ovarian cancer cells. Mol Ther 14: 164-174, 2006.

13. Ganesh S, Gonzalez-Edick M, Gibbons D, Waugh J, Van Roey M and Jooss K: Evaluation of biodistribution of a fiber-chimeric, conditionally replication-competent (oncolytic) adenovirus in CD46 receptor transgenic mice. Hum Gene Ther 20: 1201-1213, 2009.

14. Hoffmann D, Meyer B and Wildner O: Improved glioblastoma treatment with Ad5/35 fiber chimeric conditionally replicating adenoviruses. J Gene Med 9: 764-778, 2007.

15. Wakayama M, Abei M, Kawashima R, et al: E1A, E1B double-restricted adenovirus with RGD-fiber modification exhibits enhanced oncolysis for CA-deficient biliary cancers. Clin Cancer Res 13: 3043-3050, 2007.

16. Ries SJ, Brandts CH, Chung AS, et al: Loss of p14ARF in tumor cells facilitates replication of the adenovirus mutant d11520 (ONYX-015). Nat Med 6: 1128-1133, 2000.

17. Harada JN and Berk AJ: p53-Independent and -dependent requirements for E1B-55K in adenovirus type 5 replication. J Virol 73: 5333-5344, 1999.

18. Heise C, Hermiston T, Johnson L, et al: An adenovirus E1A mutant that demonstrates potent and selective systemic anti-tumoral efficacy. Nat Med 6: 1134-1139, 2000.

19. Zhang ZL, Zou WG, Luo CX, et al: An armed oncolytic adenovirus system, ZD55-gene, demonstrating potent antitumoral efficacy. Cell Res 13: 481-489, 2003.

20. Tian H, Wang J, Zhang B, et al: MDA-7/IL-24 induces Bcl-2 denitrosylation and ubiquitin-degradation involved in cancer cell apoptosis. PLoS One 7: e37200, 2012.

21. Jiang G, Liu YQ, Wei ZP, Pei DS, Mao LJ and Zheng JN: Enhanced anti-tumor activity by the combination of a conditionally replicating adenovirus mediated interleukin-24 and dacarbazine against melanoma cells via induction of apoptosis. Cancer Lett 294: 220-228, 2010.

22. Halldén G: Optimisation of replication-selective oncolytic adenoviral mutants in combination with chemotherapeutics. J BUON 14 (Suppl 1): S61-S67, 2009.

23. Saito K, Shirasawa H, Isegawa N, Shiiba M, Uzawa K and Tanzawa H: Oncolytic virotherapy for oral squamous cell carcinoma using replication-competent viruses. Oral Oncol 45: 1021-1027, 2009.

24. Khuri FR, Nemunaitis J, Ganly I, et al: A controlled trial of intratumoral ONYX-015, a selectively-replicating adenovirus, in combination with cisplatin and 5-fluorouracil in patients with recurrent head and neck cancer. Nat Med 6: 879-885, 2000. 
25. Cherubini G, Kallin C, Mozetic A, et al: The oncolytic adenovirus $\operatorname{Ad} \Delta \Delta$ enhances selective cancer cell killing in combination with DNA-damaging drugs in pancreatic cancer models. Gene Ther 18: 1157-1165, 2011.

26. Bhattacharyya M, Francis J, Eddouadi A, Lemoine NR and Halldén G: An oncolytic adenovirus defective in pRb-binding (d1922-947) can efficiently eliminate pancreatic cancer cells and tumors in vivo in combination with 5-FU or gemcitabine. Cancer Gene Ther 18: 734-743, 2011.

27. Ingemarsdotter CK, Baird SK, Connell CM, Öberg D, Halldén G and McNeish IA: Low-dose paclitaxel synergizes with oncolytic adenoviruses via mitotic slippage and apoptosis in ovarian cancer. Oncogene 29: 6051-6063, 2010.

28. Gao Y, Nankya I, Abraha A, et al: Calculating HIV-1 infectious titre using a virtual TCID(50) method. Methods Mol Biol 485 27-35, 2009.

29. Walker RA, Hanby A, Pinder SE, Thomas J, Ellis IO; National Coordinating Committee for Breast Pathology Research Subgroup: Current issues in diagnostic breast pathology. J Clin Pathol 65: 771-785, 2012.

30. Wong HH, Lemoine NR and Wang Y: Oncolytic viruses for cancer therapy: overcoming the obstacles. Viruses 2: 78-106, 2010.

31. Cun B, Song X, Jia R, et al: Combination of oncolytic adenovirus and dacarbazine attenuates antitumor ability against uveal melanoma cells via cell cycle block. Cancer Biol Ther 13: 77-84, 2012.

32. Abate-Daga D, Andreu N, Camacho-Sánchez J, et al: Oncolytic adenoviruses armed with thymidine kinase can be traced by PET imaging and show potent antitumoural effects by ganciclovir dosing. PLoS One 6: e26142, 2011.

33. Chu RL, Post DE, Khuri FR and Van Meir EG: Use of replicating oncolytic adenoviruses in combination therapy for cancer. Clin Cancer Res 10: 5299-5312, 2004

34. Menzin AW, King SA, Aikins JK, Mikuta JJ and Rubin SC: Taxol (paclitaxel) was approved by FDA for the treatment of patients with recurrent ovarian cancer. Gynecol Oncol 54: 103, 1994.

35. Flores ML, Castilla C, Ávila R, Ruiz-Borrego M, Sáez C and Japón MA: Paclitaxel sensitivity of breast cancer cells requires efficient mitotic arrest and disruption of $\mathrm{Bcl}-\mathrm{xL} / \mathrm{Bak}$ interaction. Breast Cancer Res Treat 133: 917-928, 2012.
36. Lu PH, Yu CC, Chiang PC, et al: Paclitaxel induces apoptosis through activation of nuclear protein kinase $C-\delta$ and subsequent activation of Golgi associated Cdk1 in human hormone refractory prostate cancer. J Urol 186: 2434-2441, 2011.

37. Le XF, Mao W, He G, et al: The role of p27(Kip1) in dasatinib-enhanced paclitaxel cytotoxicity in human ovarian cancer cells. J Natl Cancer Inst 103: 1403-1422, 2011.

38. Heikaus S, Matuszek KS, Suschek CV, et al: Paclitaxel (Taxol)-induced apoptosis in human epithelioid sarcoma cell lines is enhanced by upregulation of CD95 ligand (FasL/Apo-1L). J Cancer Res Clin Oncol 134: 689-695, 2008.32.

Abate-Daga D, Andreu N, Camacho-Sánchez J, et al: Oncolytic adenoviruses armed with thymidine kinase can be traced by PET imaging and show potent antitumoural effects by ganciclovir dosing. PLoS One 6: e26142, 2011.

33. Chu RL, Post DE, Khuri FR and Van Meir EG: Use of replicating oncolytic adenoviruses in combination therapy for cancer. Clin Cancer Res 10: 5299-5312, 2004

34. Menzin AW, King SA, Aikins JK, Mikuta JJ and Rubin SC: Taxol (paclitaxel) was approved by FDA for the treatment of patients with recurrent ovarian cancer. Gynecol Oncol 54: 103, 1994.

35. Flores ML, Castilla C, Ávila R, Ruiz-Borrego M, Sáez C and Japón MA: Paclitaxel sensitivity of breast cancer cells requires efficient mitotic arrest and disruption of $\mathrm{Bcl}-\mathrm{xL} / \mathrm{Bak}$ interaction. Breast Cancer Res Treat 133: 917-928, 2012.

36. Lu PH, Yu CC, Chiang PC, et al: Paclitaxel induces apoptosis through activation of nuclear protein kinase $C-\delta$ and subsequent activation of Golgi associated Cdk1 in human hormone refractory prostate cancer. J Urol 186: 2434-2441, 2011.

37. Le XF, Mao W, He G, et al: The role of p27(Kip1) in dasatinib-enhanced paclitaxel cytotoxicity in human ovarian cancer cells. J Natl Cancer Inst 103: 1403-1422, 2011.

38. Heikaus S, Matuszek KS, Suschek CV, et al: Paclitaxel (Taxol)-induced apoptosis in human epithelioid sarcoma cell lines is enhanced by upregulation of CD95 ligand (FasL/Apo-1L). J Cancer Res Clin Oncol 134: 689-695, 2008. 